\title{
Splenic macrophages in preterm infants: a necropsy study
}

\author{
S Variend, A Drummond, R Coombs
}

\begin{abstract}
Aims-To study the frequency and nature of histiocytes in the splenic red pulp of infants who died following complicated immaturity/prematurity.

Methods-Twenty four preterm/immature infants were investigated. Frozen sections of formalin fixed splenic tissue were stained with Oil Red O. Paraffin wax sections from the same tissue were conventionally stained with haematoxylin and eosin. Immunohistochemistry was carried out for a number of macrophage markers. The administration of Intralipid was compared with the presence and extent of tissue macrophages.
\end{abstract}

Results-The spleens of 10 infants showed varying degrees of Oil Red $O$ positivity ranging from mild to strong. In all these cases varying numbers of macrophages were confirmed in the splenic parenchyma in ordinary sections. The immunomarkers indicated that the histiocytes belonged to the macrophage phagocytic system. Of the 10 cases with splenic macrophages all had received Intralipid. Of those not receiving Intralipid none showed splenic macrophages. Seven had received Intralipid but did not have splenic macrophages; they had either only received small amounts of Intralipid or Intralipid was discontinued before death.

Conclusions-Splenic macrophages are common at necropsy in immature/preterm infants. The macrophages are most lucidly demonstrated using Oil Red $O$ staining in frozen sections. There is a strong association between the presence of splenic macrophages and Intralipid administration.

(f Clin Pathol 1996;49:391-394)

Department of Histopathology, Children's Hospital, Sheffield

$S$ Variend

A Drummond

Department of Neonatology, Jessop Hospital for Women, Sheffield $\mathrm{R}$ Coombs

Correspondence to: Dr S Variend, Department of Histopathology Children's Hospital, Western Bank, Sheffield S10 2TH.

Accepted for publication 23 January 1996
Keywords: splenic macrophages, preterm infants, necropsy, Intralipid.

The presence of large numbers of macrophages in human tissues is often taken to indicate the presence of a storage disorder or an infection induced state and, rarely, a malignant condition. $^{1-3}$ The finding of large numbers of macrophages in the preterm human spleen may conceivably raise similar diagnostic considerations.

An investigation of splenic tissue from 24 consecutive preterm infants using the Oil Red $\mathrm{O}$ technique showed positively staining cells in the red pulp in a significant proportion of specimens. Conventional staining of the same specimens showed that Oil Red O positivity correlates with the presence of large numbers of macrophages. The latter have been reported in the bone marrow of children ${ }^{4}$ and adults ${ }^{5}$ receiving Intralipid. Hence, we looked at the possibility of a similar association between Intralipid infusion and splenic macrophages in preterm infants.

\section{Methods}

Seventeen of the 24 infants had received intravenous Intralipid at the usually recommended dosage of up to $4 \mathrm{~g} / \mathrm{kg} /$ day, incremented from $1 \mathrm{~g} / \mathrm{kg} /$ day over four days. Gestational age ranged from 21 to 36 weeks (mean 27 weeks) and survival from less than one day to 43 days (mean eight days). Only two of the infants were assessed as small for their dates. There were 14 males and 10 females. The causes of death varied widely (table 1). Heparin had been administered in several of the cases to prevent thromboembolism complicating arterial cannulation; the dosage did not, however, exceed 30 units daily.

Postmortem examination was performed according to a standardised protocol. The spleen along with other tissues had been fixed in $10 \%$ formol-calcium for three weeks. Frozen sections were cut from formalin fixed splenic tissue and stained using the Oil Red $\mathrm{O}$ technique. Haematoxylin and eosin stained sections were studied microscopically from the same spleen. Immunohistochemistry was carried out for a number of macrophage markers using standard techniques with appropriate negative and positive controls. Diaminobenzadine was used as the chromogen. The range of antibodies included CD68, S-100 protein, lysozyme, and Mac 387. Clinical parameters, including intravenous Intralipid administration, were compared with the presence and extent of macrophage infiltration of the spleen.

Liver tissue was also stained with Oil Red $\mathrm{O}$ to assess the presence of fat-laden macrophages. These included cases in which the

\begin{tabular}{lc} 
Table 1 Causes of death & \\
\hline Cause & Number of cases \\
\hline Hyaline membrane disease & 7 \\
Immaturity & 7 \\
Bronchopulmonary dysplasia & 2 \\
Intraventricular haemorrhage and hyaline & \\
$\quad$ membrane disease & 3 \\
Intraventricular haemorrhage & 1 \\
Hypoxic encephalopathy & 1 \\
Brain malformation & 1 \\
Angiotensin deficiency & 1 \\
Hydrops fetalis & 1 \\
Total & 24 \\
\hline
\end{tabular}




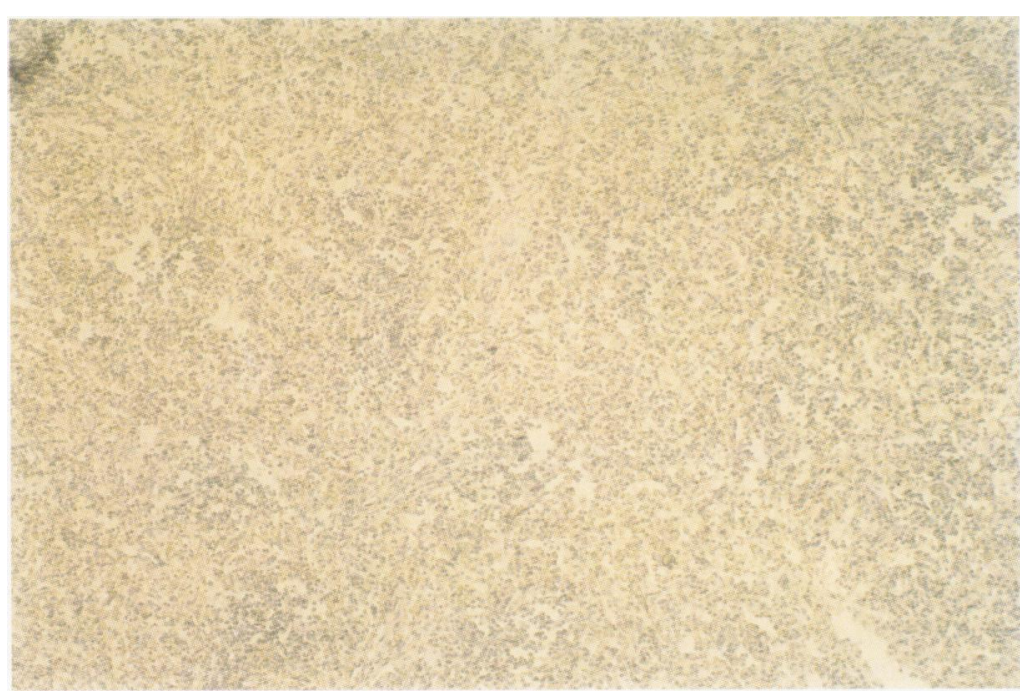

Figure 1 Oil Red $O$ staining of a section of spleen in an infant not in receipt of Intralipid showing lack of positive staining (Oil Red $O \times 135$ ).

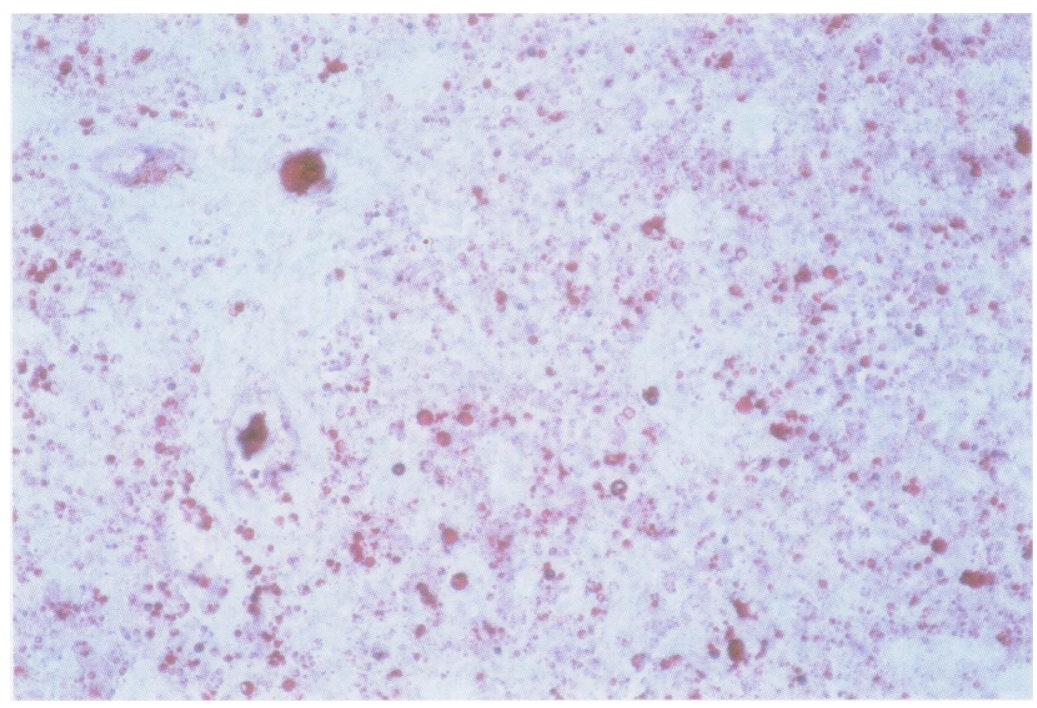

Figure 2 Oil Red $O$ staining in a section of spleen showing a strong $(+++)$ degree of positivity. Intravascular Oil Red $O$ positive material is also present (Oil Red $O \times 280)$.

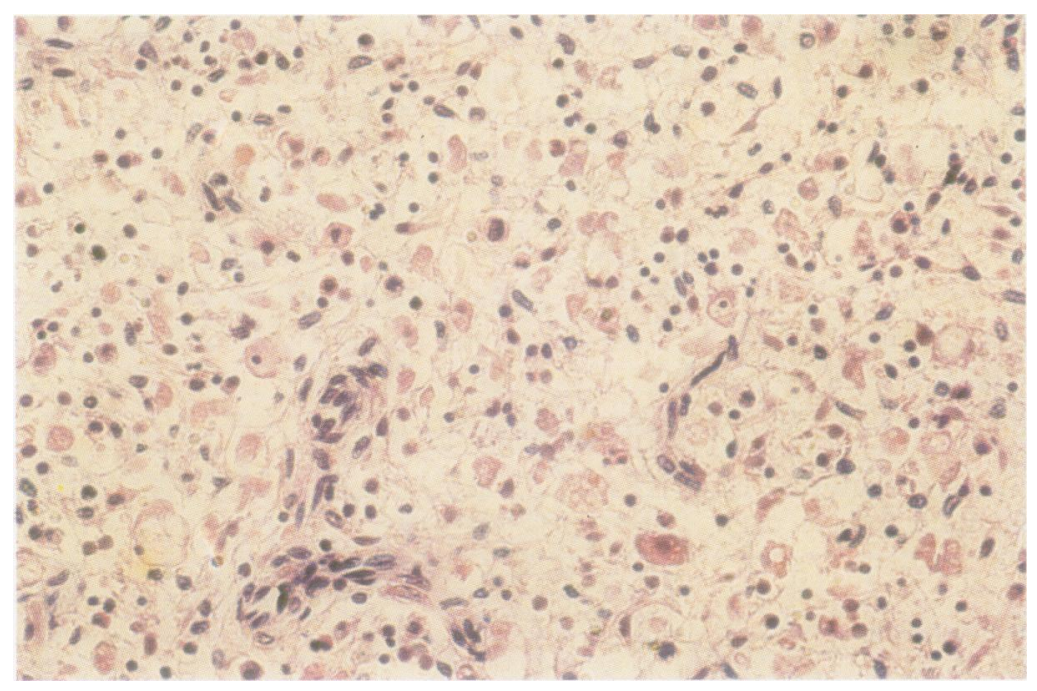

Figure 3 Conventional section from spleen showing large numbers of macrophages featuring prominent phagocytic vacuoles corresponding to strong positivity on Oil Red $O$ staining (haematoxylin and eosin $\times 558$ ).
Table 2 Relation between Oil Red $O$ positivity and Intralipid administration in the 24 infants studied

\begin{tabular}{lll}
\hline Group & Oil Red $O$ & Intralipid \\
\hline A $(n=7)$ & Negative & Negative \\
B $(n=7)$ & Negative & Positive \\
C $(n=10)$ & Positive & Positive \\
\hline
\end{tabular}

spleen was maximally involved and cases in which the spleen was negative for fat-laden macrophages despite receiving Intralipid. The bone marrow was also studied in these cases but because the tissue had been previously decalcified it was not possible to use the Oil Red O method, and only conventionally stained sections were assessed.

\section{Results}

The seven cases who had not received Intralipid did not demonstrate splenic Oil Red O positivity (group A) (fig 1). Seven cases who had received Intralipid were negative for Oil Red $\mathrm{O}$ staining in the splenic tissue (group B). Of the 10 cases with Oil Red $O$ positivity all had received Intralipid (group C) (table 2). This suggests a strong association between Oil Red $O$ positivity and the administration of Intralipid (Yates corrected $\chi^{2}=4.84, p=0.028$ ).

According to the degree of Oil Red O positivity the cases were assigned to the following categories: strong staining $(+++)$ (two cases) (fig 2); moderate $(++)$ (four cases); and mild $(+)$ (four cases).

The mean total volume of Intralipid received by group B was $25.8 \mathrm{ml}$ (range 1.3 to $72 \mathrm{ml}$ ) and the mean total volume received by group C was $152 \mathrm{ml}$ (range 7.6 to $649 \mathrm{ml}$ ). In group B the four cases who received the highest volumes had discontinued Intralipid nine to 96 hours before death and those who had received Intralipid up to the time of death had only received small volumes ( 1.3 to $6 \mathrm{ml})$. Only two cases in group $\mathrm{C}$ had Intralipid discontinued before death (six and 12 hours). One of these had received a total volume of $250 \mathrm{ml}$ and the other had received a volume of $11.5 \mathrm{ml}$; the latter infant was born after a gestational age of 23 weeks.

Among those spleens exhibiting parenchymal lipid on staining with Oil Red O, the macrophages visualised in conventional sections seemed to be actively phagocytic as shown by a coarsely vacuolated cytoplasm (fig 3 ). The phagocytosed material seemed to be limited to lipid (empty vacuoles) and did not overtly include other cellular elements such as erythrocytes or lymphocytes; however, this was sometimes difficult to exclude in the presence of postmortem changes. The splenic macrophages were positive for CD68, MAC 387 and lysozyme; they were negative for $S-100$ protein. CD68 immunochemistry gave the best results in terms of intensity and clarity of staining, which appeared undiminished by moderate degrees of postmortem autolysis.

Macrophages also appeared phagocytic in four cases from group B, but the degree of cytoplasmic vacuolation did not seem to be as 
strong as that in group C cases, and probably reflected the presence of recently deposited lipid. CD68 positive cells were easily seen in the splenic tissue from group A cases and probably reflected resting indigenous macrophages. ${ }^{6}$ They were neither as numerous nor as enlarged as the "activated" macrophages in the spleens of cases in groups B and C.

In cases in group $C$ the volume of administered Intralipid generally did not correlate strongly with the degree of Oil Red O positivity. In some of the spleens Oil Red $O$ positive material was also observed within the lumina of splenic vessels (fig 2), a feature that seemed to relate to very noticeable degrees $(+++)$ of deposited parenchymal lipid.

The hepatic portal tracts in the two group C cases contained a few fat bearing macrophages and the bone marrow displayed many foamy macrophages. By contrast, the hepatic portal triads and bone marrow were devoid of such macrophages in the seven group B cases.

\section{Discussion}

Oil Red $\mathrm{O}$ positive staining was found in a significant proportion of the spleens from preterm infants. All of the cases with Oil Red $O$ positive material had received intravenous Intralipid. The more noticeable degrees of fat deposition generally occurred with larger infusions of Intralipid, but the correlation was not strong. The immunohistochemical profile of the macrophages indicated that they belonged to the macrophage-phagocytic system. The macrophages were overtly phagocytic.

Intralipid, an emulsion of soya bean oil, egg lecithin and glycerol, is added to total parenteral nutrition which is widely used in the clinical management of ill preterm infants. It is an effective method of providing adequate calories in a small volume with a relatively low osmotic load. The fat particles of Intralipid are metabolised to chylomicrons and plasma triglycerides; the triglycerides are then hydrolysed by lipoprotein lipase to glycerol and free fatty acids. The administration of Intralipid is rarely associated with serious complications in the paediatric population. ${ }^{7}$

In group $C$ cases the volume of infused Intralipid correlated poorly with the degree of splenic Oil Red O staining. Intralipid was discontinued before death in only two cases in this group and it is likely that the lack of correlation was related to the complex metabolism of fat in the preterm infant. ${ }^{8}$ Very preterm infants and those who are small for their gestational age are also less efficient at clearing triglyceride from the plasma. ${ }^{9}$ Absence of Oil Red $O$ staining in some of the cases after discontinuation of Intralipid, as seen in group B cases, suggests that deposition of lipid in the spleen is reversible, and the relatively short intervals between the cessation of lipid infusion and the time of death in some of the cases indicate that clearing of the lipid may be rapid.

The presence of lipid within the pulmonary vascular bed of infants receiving intravenous Intralipid at the usually accepted dosage has been documented. ${ }^{10-12}$ It has been suggested that the observation represents pulmonary fat embolism complicating intravenous lipid administration ${ }^{10}$; others have expressed doubt that the phenomenon has any clinical significance. ${ }^{12}$

The fat overload syndrome, a clinical entity, is a term used to describe a haemophagocytic process complicating prolonged Intralipid administration. ${ }^{41314}$ The condition, which is typified by gastrointestinal disturbance, hepatosplenomegaly, anaemia, thrombocytopenia, coagulation disorders, and hyperlipaemia, is usually reported in adults, but children are occasionally affected. ${ }^{41314}$ Symptoms are reversible on withdrawal of lipid. The complication is rare as evidenced by its reported absence among 180 patients between birth and 21 years of age receiving Intralipid. ${ }^{7}$

The fat overload syndrome is a clinical concept and, as such, is difficult to apply to the present cases, but it is possible that the pathological observations described here are part of a continuum that includes the syndrome.

A clinical picture similar to reactive haemophagocytic syndrome has been reported in children during the course of prolonged Intralipid administration. ${ }^{4}$ Sudan-black positive macrophages exhibiting erythrophagocytosis in the bone marrow of all seven cases reported suggested hyperactivation of the phagocytemacrophage system. A relation between this condition and the fat overload syndrome is suggested as both conditions demonstrate similar haematological abnormalities.

There is ample evidence that lipid is deposited in the reticuloendothelial system after lipid infusion. Experimental animals receiving multiple infusions of fat emulsion demonstrated polarisable brown pigment within and outside the cells of the liver, spleen and bone marrow ${ }^{15}$ and intrahepatic pigment has been demonstrated in liver sections at necropsy of infants and children receiving lipid emulsion. ${ }^{1617}$ In the former study the pigment was found in the hepatocytes and the hepatic reticuloendothelial cells, and in the latter study the pigment was found in the hepatic Kupffer cells only. Hepatic and splenic foamy macrophages were also reported in an adult at necropsy after long term Intralipid infusion. ${ }^{18}$

Organs other than the spleen were not formally investigated in the current study, but a limited assessment indicated that fat containing macrophages occur concurrently in at least some extrasplenic reticuloendothelial tissues in infants receiving Intralipid. Many of the cases in the present study developed terminal sepsis and it is conceivable that the presence of a large amount of lipid occupying the reticuloendothelial system might have had a depressant effect on immune function. Whether Intralipid administration impairs host defence and facilitates infection is an ongoing debate. ${ }^{19}$ Mice pretreated with Intralipid have been found to show a greater mortality when challenged with group B streptococcus, compared with control animals. ${ }^{20}$ Moreover, infants who had received intravenous lipid emulsion were reported to be several times more likely to develop coagulase negative staphylococcal bac- 
teraemia than controls. ${ }^{21}$ Compromised immunity in the recipients of lipid emulsion may be explained in a number of ways, including functional impairment of macrophages ${ }^{22}$ and neutrophils. ${ }^{20}$ Delayed skin graft rejection was also demonstrated in rats pretreated with linoleic acid ${ }^{23}$ and oleic acid has also been used in laboratory animals to "block" the reticuloendothelial system and artificially enhance susceptibility to infection. ${ }^{24}$

1 Favara BE, McCarthy RC, Mierau GW. Histiocytosis X. Hum Pathol 1983;14:663-76.

2 Nezelof C, Barbey S. Histiocytosis: nosology and pathobiology. Pediatr Pathol 1985;3:1-41.

3 The Writing Group of the Histiocyte Society. Histiocytosis syndromes in children. Lancet 1987;i:208-9.

4 Goulet O, Girot R, Maier-Redelsperger M, Bougle D, Virelizier JL, Ricour C. Hematologic disorders following prolonged use of intravenous fat emulsions in children. $\mathcal{F}$ Parenter Enteral Nutr 1986;10:284-8.

5 MacGrath KM, Zalcberg JR, Slonim J, Wiley JS. Intralipid induced haemolysis. Br $\mathcal{F}$ Haematol 1982;50:376-8.

6 induced haemolysis. Br f Haematol $1982 ; 50: 376-8$. Lasser A. The mononuclear ph
Hum Pathol 1983;14:108-26.

7 Cohen IT, Dahms B, Hays DM. Peripheral total parental nutrition employing a lipid emulsion (Intralipid): complications encountered in pediatric patients. F Pediatr Surg 1977;12:837-45.

8 Bryan H, Shennan A, Griffin E, Angel A. Intralipid-its rational use in parenteral nutrition of the newborn. Pediatrics 1976;58:787-90.

9 Andrew G, Chan G, Schiff D. Lipid metabolism in the neonate. F Pediatr 1976;88:273-8.

10 Barson AJ, Chiswick ML, Doig CM. Fat embolism in infancy after intravenous fat emulsion. Arch Dis Child 1978;53:218-23.
11 Levene MI, Wigglesworth JS, Desai R. Pulmonary fat accumulation after Intralipid infusion in the preterm infant. Lancet 1980;ii:815-19.

12 Puntis JWL, Rushton DI. Pulmonary intravascular lipid in neonatal necropsy specimens. Arch Dis Child 1991;66: 26-8.

13 Heyman MB, Storch S, Ament ME. The fat overload syndrome. Report of a case and literature review. $A m \mathcal{J}$ Dis Child 1981;135:628-30.

14 Belin RP, Bivins BA, Jona JZ, Young VL. Fat overload with 10\% soybean oil emulsion. Arch Surg 1976;111:1391-3.

15 Tovar JA, Mahour $\mathrm{H}$, Miller SW, Isaacs $\mathrm{H}$, Smith CN. Endotoxin clearance after Intralipid infusion. 7 Pediatr Surg 1976;111:23-31.

16 Koga Y, Swanson VL, Hays DM. Hepatic "intravenous fat pigment" in infants and children receiving lipid emulsion. pigment" in infants and children

17 Paswell JH, David R, Katz Nelson D, Cohen BE. Pigment deposition in the reticulo-endothelial system after fat emulsion infusion. Arch Dis Child 1976;51:366-8.

18 Freund U, Krausz Y, Levij IS, Eliakim M. Iatrogenic lipidosis following prolonged intravenous hyperalimentation. Am f Clin Nutr 1975;28:1156-60.

19 Palmblad J. Intravenous lipid emulsions and host defensecritical review. Clin Nutr 1991;10:303-8.

20 Fischer GW, Wilson SR, Hunter KW, Mease AD. Diminished bacterial defences with Intralipid. Lancet 1980;ii: 819-21

21 Freeman J, Goldmann DA, Smith NE, Sidebottom DG Epstein MF, Platt R. Association of intravenous lipid Epstein MF, Platt R. Association of intravenous lipid in neonatal intensive care units. $N$ Engl f Med 1990;323: in neon $301-8$.

22 Strunk RC, Kunke K, Nagle RB, Payne CM, Harrison HR Inhibition of in vitro synthesis of the second (C2) and fourth (C4) components of complement in guinea pis peritoneal macrophages by a soya bean emulsion. Pediat Res 1978;13:188-93.

23 Ring J, Seifert J, Mertin J, Brendel W. Prolongation of skin allografts in rats by treatment with linoleic acid. Lancet 1974;ii: 1331 .

24 Spratt MG, Kratzing CC. Oleic acid as a depressant of reticuloendothelial activity in rats and mice. 7 Reticuloendothel Soc 1975;17:135-40. 